Meta

Journal des traducteurs

Translators' Journal

\title{
Analyse sémantique comparative des structures des vocabulaires scientifiques anglais et français
}

\section{Christine Bagge}

Volume 28, numéro 4, décembre 1983

URI : https://id.erudit.org/iderudit/001943ar

DOI : https://doi.org/10.7202/001943ar

Aller au sommaire du numéro

Éditeur(s)

Les Presses de l'Université de Montréal

ISSN

0026-0452 (imprimé)

1492-1421 (numérique)

Découvrir la revue

Citer cet article

Bagge, C. (1983). Analyse sémantique comparative des structures des vocabulaires scientifiques anglais et français. Meta, 28(4), 391-399.

https://doi.org/10.7202/001943ar d'utilisation que vous pouvez consulter en ligne. 


\section{ANALYSE SÉMANTIQUE COMPARATIVE DES STRUCTURES DES VOCABULAIRES SCIENTIFIQUES ANGLAIS ET FRANÇAIS}

\section{INTRODUCTION}

La terminologie, encore relativement récente en tant que discipline, est avant tout une activité pratique qui a pour but immédiat de résoudre des problèmes d'expression et de communication. C'est pour cette raison qu'il est difficile de la faire rentrer dans un cadre théorique. Cependant, les termes sont exprimés par des signes qui appartiennent au langage, et au cours des dix dernières années nombreux ont été ceux qui ont essayé de trouver la place de la terminologie au sein du domaine de la linguistique appliquée (Guilbert, 1975; Wüster, 1975; Auger, 1975; Rey, 1977; Kocourek, 1978). La tentative la plus récente et la plus complète dans ce domaine est l'œuvre théorique de $\mathbf{R}$. Kocourek (1982).

Il est donc maintenant reconnu que la terminologie doit beaucoup à la linguistique, en particulier à la sémantique. En fait, l'unité terminologique est totalement définie au sein de la sémantique. Selon R. Kocourek (1978: 220) : «[...] the essential characteristic of terms seems to lie in the area of terminological semantics. The term has variously been defined as definite and precise, unambiguous and unique, as being the designation of a concept, as having a specifically defined meaning. All these features are semantic in nature.» Ce phénomène avait déjà été relevé en 1959 par le père de la terminologie, E. Wüster, qui écrivait alors : «Les dictionnaires techniques doivent être présentés suivant un ordre sémantique et non alphabétique.» (cité dans Mounin, 1980 : 130). Cette analyse portera donc sur l'aspect sémantique du vocabulaire scientifique. Bien que la sémantique ait été exclue du domaine de la linguistique par des Américains tels que L. Bloomfield et qu'elle soit encore souvent considérée comme la discipline la moins scientifique de la linguistique, elle a été réhabilitée. J.R. Firth (1968: 87), qui a toujours réagi contre cette tendance anti-sémantique, cite Bloch avec ferveur : «[...] meaning [...] is so obviously useful [...] that any linguist who refused to employ it would be largely wasting his time.»

Plus précisément, cette recherche essaiera de comparer les vocabulaires scientifiques français et anglais en mettant à profit les méthodes mises à notre disposition par la sémantique structurale qui suit la tradition saussurienne (Lyons, 1981 : 230-235). Elle tiendra compte en particulier des deux énoncés suivants : «There can no longer be any doubt that [...] Trier's paradigmatic relations must be incorporated in any satisfactory theory of lexical structure» (Lyons, $1981: 261$ ) et « [...] field-theory has proved its worth as a general guide for research in descriptive semantics over the last forty years» (Lyons, 1981: 267). La sémantique structurale telle qu'elle s'est développée en France s'est concentrée, comme le remarque J. Lyons (1981:267) d'une part sur les parties du vocabulaire d'une langue sujettes à des changements rapides (Matoré, 1953) et d'autre part sur l'analyse stylistique de textes et sur d'autres systèmes sémiotiques que les langues (Greimas, 1965; Barthes, 1964). Puisqu'une des caractéristiques les plus typiques des termes scientifiques est leur constance 
de forme et de sens, et que cette analyse s'intéresse aux termes et non pas aux textes scientifiques, cette perspective française ne sera pas pertinente à cette recherche.

\section{MÉTHODOLOGIE}

Les organisations qui se spécialisent en terminologie effectuent deux types de recherche selon les besoins de leurs clients : ponctuelle ou thématique. La recherche ponctuelle traite des termes isolés et pour cette raison n'est pas aussi fiable (Auger et Rousseau, 1978: 63). La recherche thématique, par contre, produit des lexiques exhaustifs qui couvrent de vastes secteurs des vocabulaires scientifiques et techniques. Lorsque l'on traite ainsi le vocabulaire en tranches homogènes, il est possible de déterminer la structure conceptuelle spécifique à chaque langue dans un domaine donné et ensuite de les comparer et d'établir où ces structures ne coïncident pas. Chaque terme doit son sens au réseau qu'il forme avec les autres termes du même domaine que l'on nomme «champ sémantique». Ce terme décrit en linguistique une notion assez vague et il existe probablement autant d'interprétations de cette notion que de linguistes qui l'ont étudiée. O. Duchacek (1960) les a inventoriées et situées les unes par rapport aux autres. Mais les définitions suivantes s'appliquent à la majorité d'entre elles: "aire couverte, dans le domaine de la signification, par un groupe de mots de la langue» (Dubois et al., 1973) et «a group of words closely related in meaning, often subsumed under a general term» (Lehrer, 1974: 1).

Une fois établi le fait qu'il est nécessaire de traiter les termes sous forme de champs sémantiques, il reste à déterminer l'envergure de ces champs. Un terminologue ferait normalement l'étude complète d'un domaine scientifique donné - une branche de la biologie par exemple. Cependant, cette analyse a pour ambition d'essayer de tirer des conclusions sur le vocabulaire scientifique pris dans son ensemble, non pas spécifiquement sur le vocabulaire d'une seule science. Il s'agira donc de considérer un échantillon, pris au hasard, de concepts scientifiques appartenant à diverses sciences et d'étudier chaque concept de façon exhaustive.

Pour des raisons pratiques, il n'est cependant pas possible de relever au hasard un échantillon de concepts scientifiques. La méthodologie consistera donc, tout d'abord, à former un corpus de termes scientifiques pris au hasard dans un dictionnaire scientifique général. Grâce à une analyse componentielle sommaire, ces termes seront replacés dans leur contexte et les concepts auxquels ils appartiennent seront ainsi identifiés. Quand une analyse componentielle est effectuée dans le domaine de la terminologie, le terme 'descripteurs' (Dubuc, 1978: 23) est généralement utilisé au lieu de 'traits de sens' ou 'sèmes'. Ces descripteurs seront extraits de la définition du terme donnée par les spécialistes du domaine. Ensuite tous les termes appartenant à chaque concept seront regroupés dans chacune des deux langues. L'établissement de l'échantillon tel qu'il vient d'être décrit correspond à ce que $H$. Blalock $(1960: 405)$ nomme «a single-stage cluster design» et pourrait être représenté schématiquement tel que dans la figure 1 . 
Figure 1

Établissement de l'échantillon

\begin{tabular}{ll}
\hline $\begin{array}{l}\text { Terme } \\
\text { initial } \\
\text { pris au } \\
\text { hasard }\end{array}$ & $\begin{array}{l}\mathrm{X} \text { termes anglais } \\
\text { appartenant à ce concept, } \\
\text { classés hiérarchiquement }\end{array}$ \\
& $\begin{array}{l}\mathrm{X} \text { termes français } \\
\text { appartenant à ce concept, } \\
\text { classés hiérarchiquement }\end{array}$ \\
\hline
\end{tabular}

Étant donné qu'une science est un système de concepts explicitement organisés, le champ sémantique sera délimité par la définition du concept fournie par les spécialistes du domaine en question. D'autre part, il comprendra les unités terminologiques situées sur l'axe paradigmatique et reliées entre elles par l'hyponymie, une des relations de sens fondamentale dans la structuration du vocabulaire (Lyons, 1981: 291), particulièrement dans le domaine scientifique (Germain et Leblanc, 1982: 49). Cette relation paradigmatique impose au vocabulaire une structure hiérarchique qui sera représentée schématiquement par un arbre hiérarchique pour chaque concept dans chaque langue. Étant donné que la classe lexicale principale en terminologie est la classe nominale (Maillot, 1969: 78; Kocourek, 1982: 170), cette recherche ne traitera que des termes nominaux (noms ou syntagmes nominaux). Il est naturellement indispensable d'étudier les structures du vocabulaire scientifique de chaque langue séparément avant de les comparer.

\section{RESULTATS (voir table 1)}

Cette analyse a pris pour point de départ 34 termes initiaux relevés au hasard dans un dictionnaire scientifique (Lapedes, 1978). Ces termes appartiennent à différents domaines tels que la chimie, la physique, l'astronomie, la biologie, la géologie. Une analyse componentielle sommaire de ces 34 termes a permis de déceler les 34 concepts auxquels ils appartiennent respectivement (voir appendice). Un arbre hiérarchique paradigmatique a été établi pour chacun de ces concepts indépendamment dans les deux langues. Ces données ont été établies avec l'aide d'étudiants de troisième année de l'École des traducteurs de l'Université Laurentienne. Finalement, pour chaque paire d'arbres, une comparaison de l'arbre anglais avec l'arbre français a été effectuée et a permis de constater que 11 paires, soit $32 \%$ de l'échantillon total, présentaient certaines dissimilitudes qui se classent en trois catégories.

Tout d'abord, dans ce contexte, on entend par «case vide» l'absence d'un terme d'une place particulière dans un arbre d'une des deux langues lorsque ce terme est présent dans l'arbre correspondant de l'autre langue. Alors qu'aucun cas de ce phénomène ne se trouvait dans les arbres en anglais, probablement parce que l'échantillon étudié n'est pas très important, 18 cases vides ont été décelées dans les arbres en français. Comme il n'est pas possible de les citer toutes, deux exemples représentatifs suffiront.

La comparaison des deux sections d'arbres suivantes (voir figure 2), qui font partie d'une classification générale des bactéries, montre que le français 
n'a pas de terme superordonné qui réunisse «tréponèmes» et «spirilles» alors qu'en anglais ce terme existe.

Figure 2

Sections de la paire d'arbres hiérarchiques des bactéries
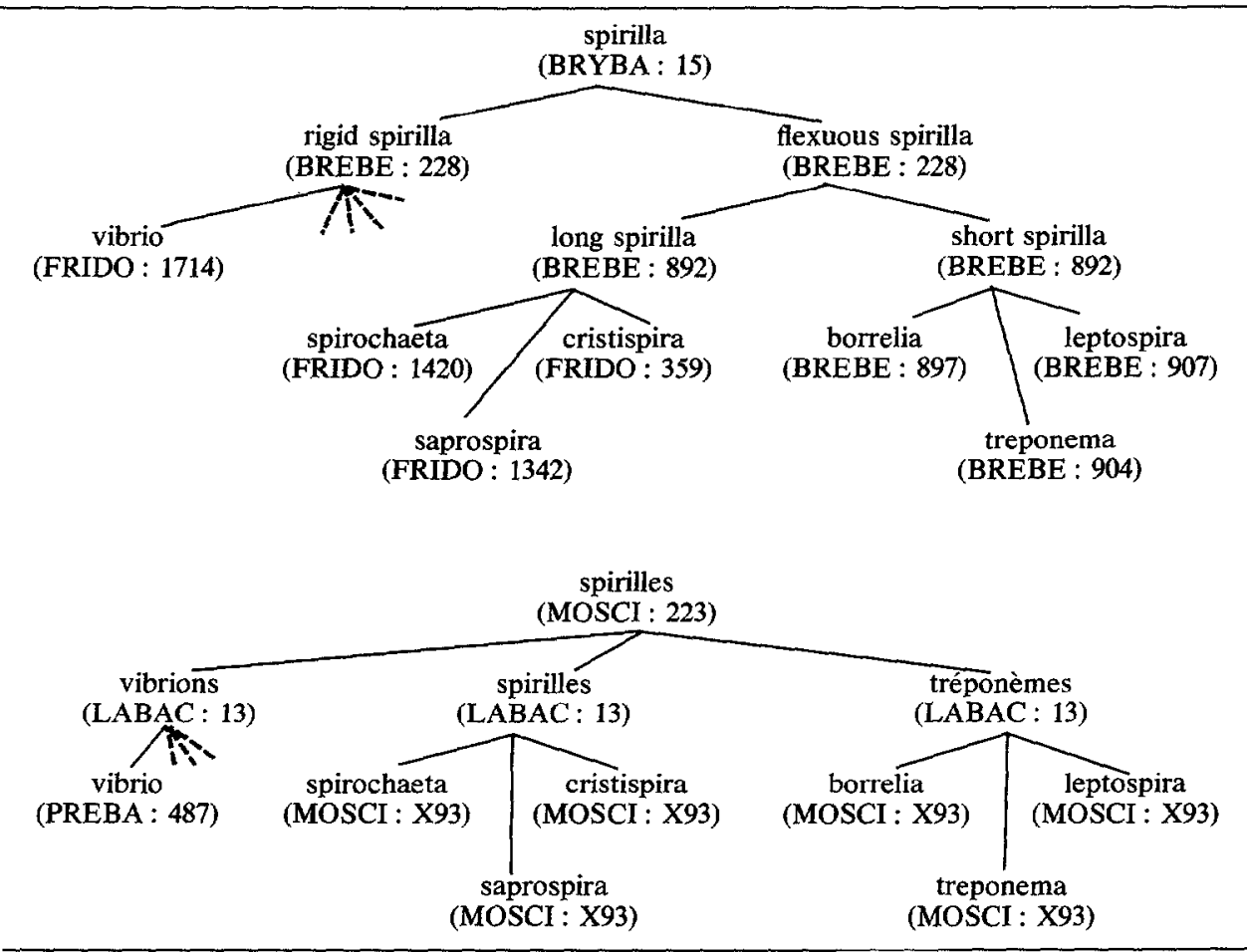

Un examen de ces deux sections d'arbres révèle que cette case vide est due à une différence dans le choix des termes utilisés pour désigner la même réalité, autrement dit au fait que les deux langues ne découpent pas la réalité de la même façon. En anglais les spirilles sont classés selon certaines de leurs caractéristiques complémentaires (rigid - flexuous; long - short). En français, bien que ces caractéristiques ne soient pas ignorées puisqu'on a les mêmes groupes cohyponymes, ces cohyponymes ont généralement pour superordonné le plus important d'entre eux (spirilles $\rightarrow$ spirilles; vibrio $\rightarrow$ vibrions; treponema $\rightarrow$ tréponèmes).

Le second exemple est emprunté au domaine de la physique nucléaire. Bien que les réactions nucléaires puissent être «spontanées» ou «provoquées» en français de la même façon qu'elles sont «spontaneous» ou «induced» en anglais, les textes scientifiques écrits en français ne font pas la distinction entre les deux de façon explicite. Autrement dit, à moins que le texte ne spécifie qu'il s'agit d'une «fission spontanée», le terme «fission» fait référence à une «fission provoquée». Ceci est probablement dû au fait que c'est avant tout de la fission provoquée que l'on s'occupe aujourd'hui à cause de ses multiples 
utilisations possibles. Ce phénomène aura des conséquences sur la structure du vocabulaire de ce domaine, comme le montre la figure 3.

Figure 3

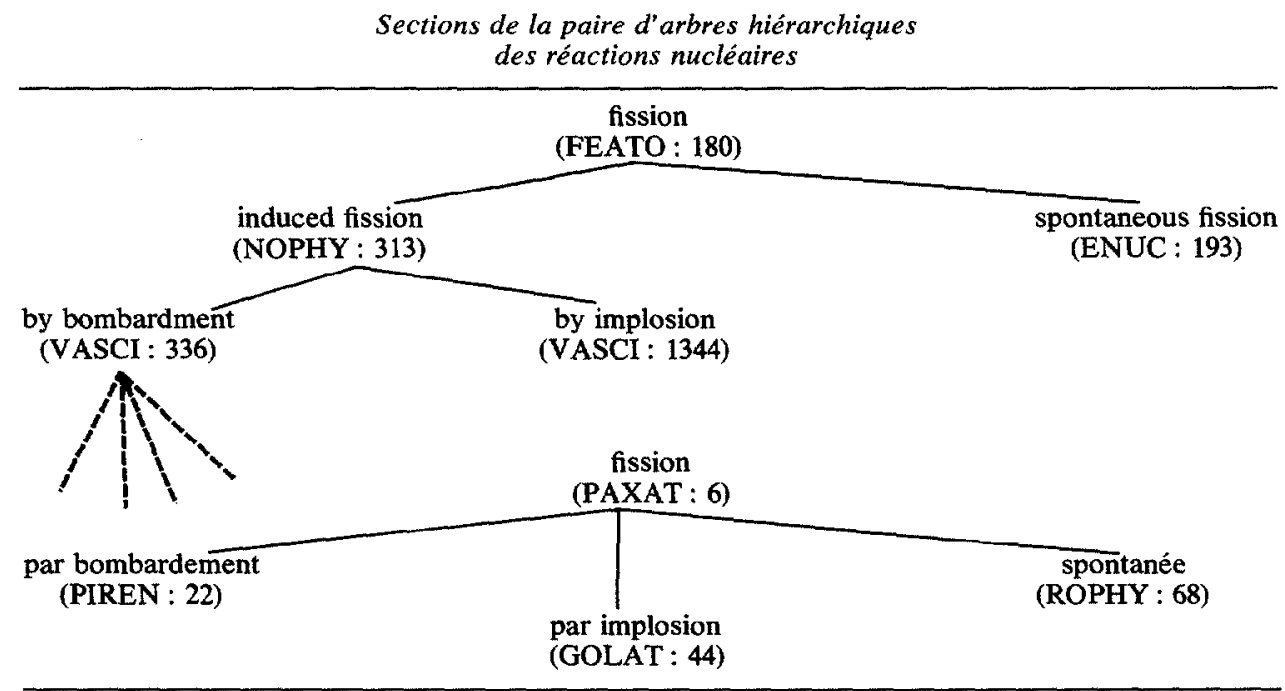

La deuxième catégorie de dissimilitudes que l'on a trouvée dans la structure des vocabulaires scientifiques des deux langues est le reflet d'une variation dans la logique organisationnelle de ces deux langues qui mène à différents systèmes de classification. On a relevé six cas de ce phénomène dans l'échantillon étudié. En anglais, par exemple, les glandes exocrines sont classées d'après leur forme et leur structure. Une telle classification pourrait exister en français, mais les scientifiques qui s'expriment dans cette langue ont préféré choisir un autre critère - la place de ces glandes dans le corps (voir figure 4).

De la même façon, les polyosides en anglais sont classés selon leur structure, alors qu'en français le critère utilisé est l'endroit où on les trouve (cf. figure 5).

Finalement, il peut arriver que les deux langues appliquent différemment un système de classification qui se fait selon les mêmes critères. Autrement dit, bien que les superordonnés soient équivalents, les cohyponymes ne sont pas toujours les mêmes. La paire d'arbres hiérarchiques des bactéries est un bon exemple de ce phénomène, le seul que l'on ait trouvé dans l'échantillon étudié. Le français est plus strict que l'anglais lorsqu'il s'agit de décider quelles espèces répondent aux critères caractéristiques d'une certaine famille. Dans le système français, qui offre moins de variantes que le système anglais, il y a plus de termes qui sont considérés comme incertains du point de vue de leur classification. D'autre part, un grand nombre de bactéries se classent en anglais dans la catégorie des bacilles alors qu'en français ils appartiennent au groupe des cocci. Ceci s'explique facilement par le fait que ces bactéries sont pléomorphiques et que le choix de leur place dans l'arbre est souvent arbitraire. 
Figure 4

Sections de la paire d'arbres

hiérarchiques des glandes

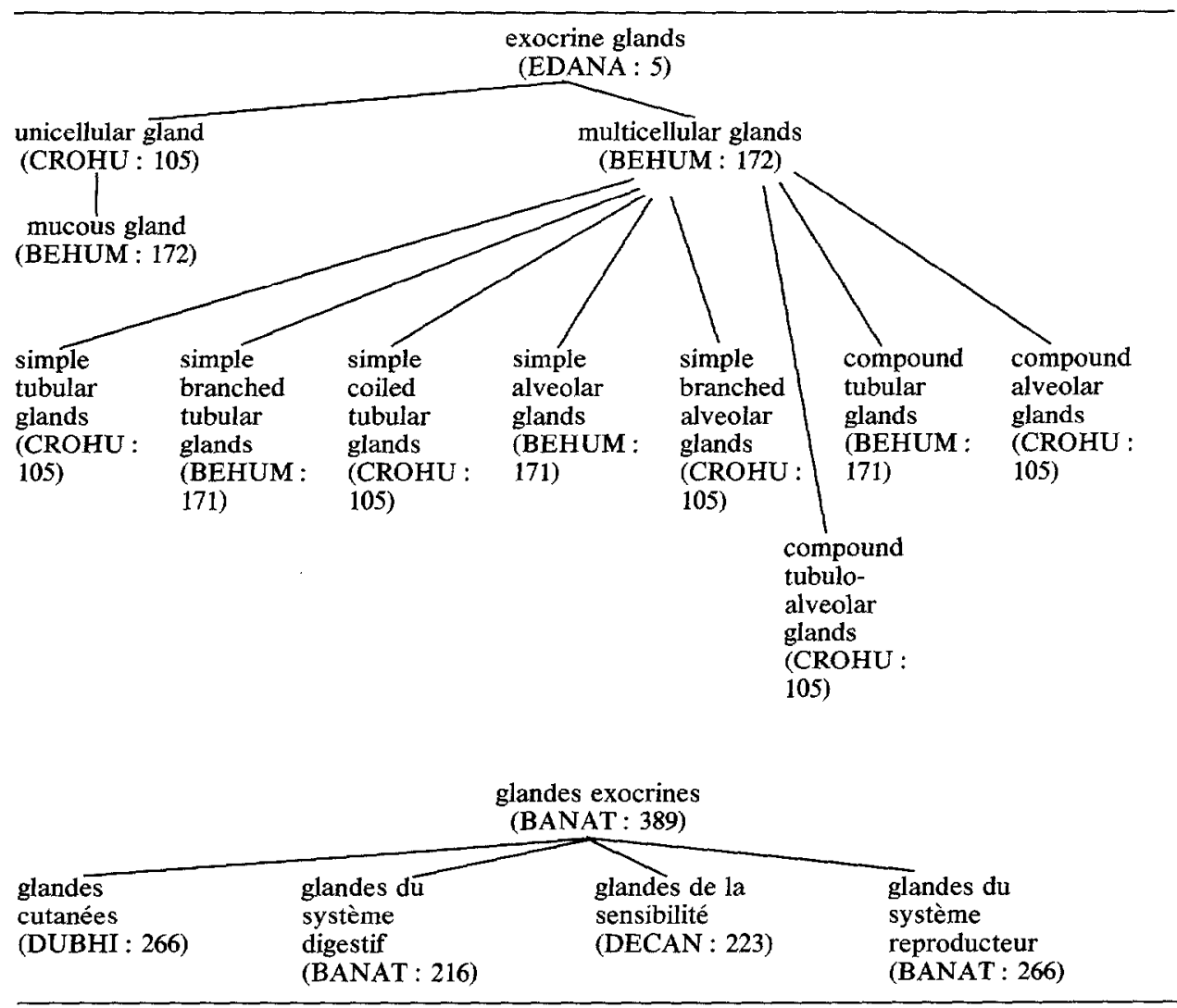

Figure 5

Sections de la paire d'arbres

hiérarchiques des macromolécules

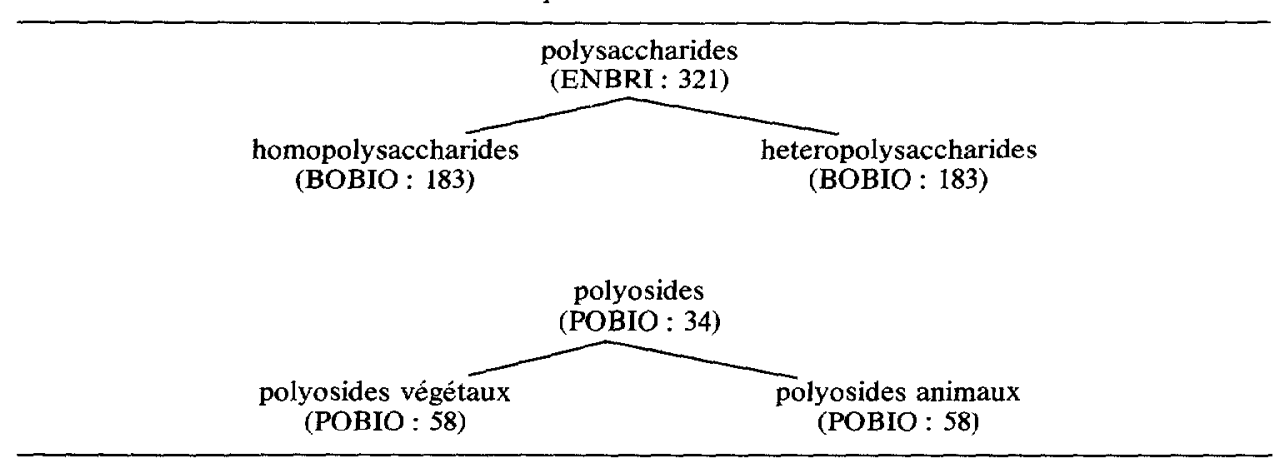


Table 1

Résumé des résultats

Nombre de termes initiaux $=34$

Nombre de concepts $=34$

Nombre de paires d'arbres $=34$

\begin{tabular}{c|c|c|c|c}
\hline \multicolumn{3}{c|}{$\begin{array}{c}\text { Nombre de paires d'arbres qui } \\
\text { présentent des différences } \\
11(32 \%)\end{array}$} & $\begin{array}{c}\text { Nombres de paires } \\
\text { d'arbres similaires } \\
23\end{array}$ \\
\hline \multirow{2}{*}{$\begin{array}{c}\text { Cases } \\
\text { vides }\end{array}$} & $\begin{array}{c}\text { Différents } \\
\text { systèmes de } \\
\text { classification }\end{array}$ & $\begin{array}{c}\text { Applications } \\
\text { différentes }\end{array}$ & \\
\hline
\end{tabular}

\section{CONCLUSION}

Bien que n'utilisant qu'un échantillon restreint, cette analyse a donné des résultats concluants. Tout d'abord, elle a montré encore une fois que la linguistique peut fournir des outils précieux lorsqu'il s'agit de comparer les terminologies de différentes langues. D'autre part, elle a confirmé l'hypothèse de départ : bien qu'une des caractéristiques les plus importantes de la terminologie scientifique soit le fait que ses structures linguistiques dépendent de la réalité non linguistique qu'est celle des sciences, ces structures varient d'une langue à l'autre et ne sont pas toujours aussi internationales qu'on le pense souvent.

Christine Bagge

Appendice

Termes

1. acetolysis

2. band spectrum

3. black hole

4. calcium chloride

5. cell

6. cistron

7. conjunction

8. dew point

9. ductless glands

10. equilibrium

11. fermions

12. fossil fuels

13. Gegenschein (counter glow)

14. ground waves (direct waves)
Concepts

chemical reactions

spectra

regions/objects in

space

compounds of calcium

cells

units of genetic

information

configurations of

celestial bodies

significant points

on the temperature

scale

glands

states existing

between forces or

effects

elementary particles

fuels

light phenomena

seen in the sky

waves
Domaine

chimie

physique

astronomie

chimie

biologie

biologie

astronomie

physique

biologie

physique

physique

chimie

astronomie

physique 

15. hormone
16. implosion
17. isocline
18. levorotatoric substances
19. light-year
20. manometer
21. nadir
22. nuclear reaction
23. osmosis
24. period
25. piezoelectric effect
26. quantum
27. reactance
28. ribonucleic acid (RNA)
29. semiconductor
30. sodium azide
31. spirillum
32. sulphide
33. testosterone
34. topaz

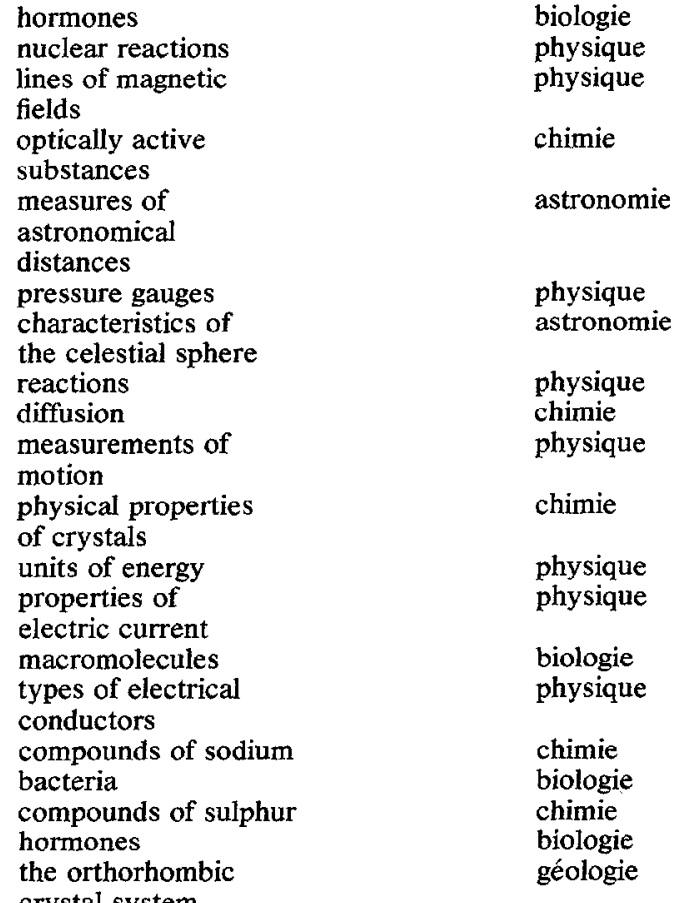

AUGER, P. (1975) : la Terminologie: une discipline linguistique du XXe siècle. Dans Essai de définition de la terminologie, $\mathrm{p}$. 59-71.

AUGER, P. et L.-J. ROUSSEAU (1978): Méthodologie de la recherche terminologique, Québec, Office de la langue française.

BARTHES, R. (1964) : Éléments de Sémiologie, Paris, Seuil.

BLALOCK, H. (1960): Social Statistics, New York, McGraw-Hill.

DUBOIS, J. et al. (1973) : Dictionnaire de linguistique, Paris, Larousse.

DUBUC, R. (1978) : Manuel pratique de terminologie, Montréal, Linguatech.

DUCHACEK, O. (1960) : «Les champs linguistiques $»$, Philologica Pragensia, 3, p. 22-35.

Actes du Colloque international de terminologie (1975) : Essai de définition de la terminologie, Québec, Régie de la langue française.

FIRTH, J.R. (1968) : "Linguistics and Translation", Selected Papers of J.R. Firth 195259 , Bloomington and London, Indiana University Press.

GERMAIN, C. et Raymond LEBLANC (1982) : la Sémantique, Montréal, Les Presses de l'Université de Montréal.

GREIMAS, A. (1965) : la Sémantique structurale, Paris, Larousse.

GUILBERT, L. (1975): «Terminologie et linguistique», dans Essai de définition de la terminologie, p. 13-26.

KOCOUREK, R. (1978) : «Prerequisites for an Applicable Linguistic Theory of Terminology», Proceedings of the $5^{\text {th }}$ Congress of l'Association internationale de linguistique appliquée, Montréal, Les Presses de l'Université Laval, p. 216-228.

KOCOUREK, R. (1982) : la Langue française de la technique et de la science, Paris, La documentation française.

LAPEDES, D.N. Éditeur (1978) ; Dictionary of Scientific and Technical Terms, New York, McGraw-Hill ( $2^{e}$ édition).

LEHRER, A. (1974): Semantic Fields and Lexical Structure, New York, American Elsevier.

LYONS, J. (1981): Semantics (1 and 2), Cambridge, Cambridge University Press.

MAILLOT, J. (1969) : la Traduction scientifique et technique, Paris, Éditions Eyrolles.

MATORÉ, R. (1953) : la Méthode en lexicologie, Paris, Didier.

MOUNIN, F. (1980): les Problèmes théoriques de la traduction, Saint-Amand, Gallimard. 
REY, A. (1977) : «Définition de la terminologie en tant que discipline linguistique autonome: état de la question", dans Actes du 6e colloque international de terminologie, p. 229-257.

WÜSTER, E. (1975) : «La théorie générale de la terminologie - un domaine interdisciplinaire impliquant la linguistique, la logique, l'ontologie, l'informatique et les sciences des objets, dans Essai de définition de la terminologie, p. 49-57.

Bibliographie des figures

Figure 2

BREBE BREED, R.S. et al. (1957): Bergey's Manual of Determinative Bacteriology, New York, The Williams and Wilkins Press.

BRYBA BRYAN, A.H. et al. (1968): Bacteriology, New York, Barnes and Noble.

FRIDO FRIEL, J.P. (1974): Dorland's Illustrated Medical Dictionary, Philadelphia, W. B. Saunders.

LABAC LAVERGNE, E. (de) (1966) : les Bactéries, Paris, Presses universitaires de France. MOSCI MORVAN, R.G., éditeur (1971): Encyclopédie internationale des sciences et des techniques, Paris, Presses de la cité.

PREBA PREVOT, A.R. et al. (1967): les Bactéries anaérobies, Paris, Dunod.

Figure 3

ENUC ENGE, H.A. (1966): Introduction to Nuclear Physics, Don Mills, AddisonWesley.

FEATO FEINBERG, J.G. (1960): The Story of Atomic Theory and Atomic Energy, New York, Dover Publications.

GOLAT GOLDSCHMIDT, B. (1962): l'Aventure atomique, France, Librairie Arthème Fayard.

NOPHY NORWOOD, J. Jr. (1976): Twentieth-Century Physics, New Jersey, PrenticeHall.

PAXAT L'atome pour ou contre l'homme (1958) : Paris, Éditions Pax Christi.

PIREN PIRAUX, H. (1959): Petit lexique de l'énergie atomique, Paris, Éditions Eyrolles.

ROPHY ROSSEL, J. (1970): Physique générale, Neuchâtel, Éditions du Griffon.

VASCI Van Nostrand's Scientific Encyclopedia (1976) : New York, Van Nostrand Reinhold ( $5^{\mathrm{e}}$ édition).

Figure 4

BANAT BASMAJIAN, J. (1977): Anatomie, Paris, Maloine ( $7^{\mathrm{e}}$ édition).

BEHUM BECK, W.S. (1971): Human Design, New York, Harcourt Brave Jovanovich.

CROHU CROUCH, J. et R. MCCLINTIC (1976): Human Anatomy and Physiology, Toronto, John Wiley and Sons.

DECAN DÉCARIE, A. (1975) : Anatomie et physiologie humaine, Montréal, HRW.

DUBHI DUBREUIL, G. et A. BAUDRIMONT (1959): Manuel théorique et pratique d'histologie, Paris, Vigot-Frères.

EDANA EDWARDS, L.F. et G. GAUGHRAN (1971) : Concise Anatomy, New York, McGraw-Hill.

Figure 5

BOBIO BOHINSKI, R.C. (1973) : Modern Concepts in Biochemistry, Boston, Allyn and Bacon.

POBIO POLONOVSKI, M. et al. (1959) : Biochimie médicale, Paris, Masson et cie.

ENBRI Encyclopadia Britannica (vols. 15 et 16) (1971), Chicago, Encyclopædia Britannica.

\section{ENRICHISSEMENT DU VOCABULAIRE DE L'AUDIOVISUEL ET DE LA PUBLICITÉ}

Accroche (n.f.).

Domaine : Publicité.

Définition : Partie d'une annonce publicitaire attirant l'attention, généralement le titre.

Anglais : Catching.
Achat groupé (n.m.).

Domaine : Audiovisuel (général).

Définition : Achat d'un ensemble de programmes à un même vendeur.

Anglais : Package. 
Aguichage (n.m.).

Domaine : Publicité.

Définition : Procédé publicitaire qui utilise Anglais : Teasing.

Aguiche (n.f.).

Domaine : Publicité

Définition : Phase initiale d'une campagne publicitaire se présentant sous forme d'énigme destinée à inciter et à maintenir l'at-

Anglais : Teaser. tention du public.

Amplificateur de sonorisation (n.m.)

Domaine : Audiovisuel/Son.

Définition : Amplificateur de puissance destiné à la sonorisation d'un local ou d'un espace important.

Note

: Le terme «amplificateur de public address » ne doit pas être employé.

Anglais : Public address-amplifier.

Ampli-Syntoniseur (n.m.).

Domaine : Audiovisuel/Son.

Définition : Élément de chaîne électroacoustique intégrant simultanément la fonction de syntoniseur et celle d'amplificateur audiofréquence.

Note : Le terme «ampli-tuner» ne doit Anglais : Receiver. pas être employé.

Archives.

Voir Images d'archives.

Baladeur (n.m.)

Domaine : Audiovisuel/Son.

Définition : Appareil portatif de reproduction sonore et éventuellement d'enregistrement, que l'on peut utiliser en se déplaçant et dont l'écoute s'effectue au moyen d'un casque léger.

Note : Le terme «walkman", qui est une marque déposée, ne doit pas être utilisé.

Baladeur radio (n.m.).

Domaine : Audiovisuel/Son.

Définition : Récepteur radio portatif que l'on peut utiliser en se déplaçant, et dont l'écoute s'effectue au moyen d'un casque léger.

Bande-mère.

Voir Souche.

Bande promo.

Voir Bande vidéo promotionnelle.

Bande vidéo (n.f.)

Domaine : Audiovisuel/Télévision-Vidéo.
Définition : Bande magnétique servant à l'enregistrement des images et du son.

Anglais : Video-tape.

Bande vidéo promotionnelle (n.f.) (Abréviation: Bande promo [n.f.], Promo [n.f.]).

Domaine : audiovisuel (général), publicité

Définition : court programme réalisé à l'aide de moyens techniques perfectionnés, pour mettre en

Anglais : Video-clip. valeur un sujet donné.

Base de campagne (n.f.).

Domaine : Publicité

Définition : Ensemble de réflexions qui permet de passer à la réalisation d'une campagne publicitaire.

Anglais : Copy platform.

Câbleur.

Voir Câbliste.

Câblier.

Voir Câbliste.

Câbliste (n.m.).

Domaine : Audiovisuel/cinéma, télévision.

Définition : Personne chargée de manipuler les câbles d'une caméra lors de ses déplacements dans une prise de vue.

Note : Différent du «câbleur», qui effectue les montages électriques, et du «câblier», qui

Anglais : Cableman fabrique ou pose les câbles.

Cadreur (n.m.)

Synonyme: Opérateur de prises de vues (n.m.).

Domaine : Audiovisuel/Télévision.

Définition : Personne chargée du maniement d'une caméra, de la mise au point, ainsi que de la définition d'un champ de prises de

Anglais : Cameraman. vues pour composer l'image.

Carton.

Voir Carton publicitaire.

Carton publicitaire (n.m.). (Abréviation : Carton [n.m.]).

Domaine : Publicité

Définition : Matériel publicitaire destiné à

Anglais

la vitrine ou à l'étalage.

Ciné-parc (n.m.).

Domaine : Audiovisuel/Cinéma.

Définition : Cinéma de plein air où le spectateur assiste à la projection assis dans sa voiture.

Anglais :Drive-in cinema.

Code temporel (n.m.).

Domaine : Audiovisuel/Télévision-vidéo. 
Définition : Code électronique permettant le repérage du temps sur une bande vidéo.

Anglais : Time-code.

Commanditaire (n.m.).

Domaine : Publicité. physique ou morale

Définition : Personne physique ou morale qui soutient l'organisation cuerement ou au moyen de prestations de services, afin d'obtenir la contrepartie des Anglais : Sponsor. effets publicitaires.

Commanditer (v.tr.).

Synonyme : Parrainer (v.tr.).

Dérivé : Commanditaire (n.m.)

Domaine : Publicité.

Définition : Soutenir l'organisation d'une manifestation, financièrement ou au moyen de prestations de services, afin d'obtenir la contrepartie des effets publicitaires.

Note : Le terme «sponsoriser» ne doit Anglais : To sponsor. pas être employé

Conducteur (n.m.).

Domaine : Audiovisuel/Télévision, Vidéo, Son.

Définition : Liste écrite ou informatisée donnant l'ordre chronologique des séquences d'un programme audiovisuel.

Contour (n.m.).

Synonyme : Correction physiologique (n.f.).

Domaine : Audiovisuel/Son.

Définition : Sur un amplificateur de hautefidélité, dispositif permettant de relever automatiquement le niveau des fréquences graves et aiguës lorsque le niveau du volume sonore est faible.

Anglais : Loudness.

Copie d'antenne (n.f.).

Domaine : Audiovisuel/Télévision, Son.

Définition : Copie d'un enregistrement sur film ou sur bande magnétique, spécifiquement destinée à la diffusion par un émetteur de radiodiffusion sonore ou de télévision.

Anglais : air print, release print.

Copie de diffusion (n.f.).

Domaine : Audiovisuel (général).

Définition : Copie d'un enregistrement sur film ou sur bande magnétique, spécifiquement destinée à la diffusion, quel qu'en soit le moyen.

Correction physiologique.

Voir Contour.
Couper sec.

Voir Sec.

Crayonné (n.m.).

Synonyme : Esquisse (n.f.).

Domaine : Publicité/Presse.

Définition : Avant-projet donnant une idée première de la réalisation publicitaire envisagée.

Anglais : Simple lay-out, rough lay-out, rough.

Débordement (n.m.).

Domaine : Publicité

Définition : Partie de la diffusion publicitaire dont la zone de couverture déborde du territoire prévu

Anglais : Overlap par le plan de campagne.

Désynchronisation ponctuelle (n.f.).

Domaine : Audiovisuel/Vidéo.

Définition : Décalage du son par rapport à l'image en un point de montage.

Anglais : Audio-video-split.

Disque audionumérique (n.m.).

Synonyme : Mini-disque (n.m.)

Domaine : Audiovisuel/Son.

Définition : Disque sur lequel le signal audiofréquence est enregistré sous forme de signaux numériques.

Note : Le terme «compact disc», qui est une marque déposée, ne doit pas être utilisé.

Distribution artistique (n.f.).

Domaine : Audiovisuel/Cinéma, Télévision, Son: Publicité.

Définition : 1. Recherrhe des artistes et attribution des rôles.

2. Ensemble des interprètes d'une œuvre.

Anglais : 1. Casting.

2. Cast.

Document (n.m.)

Domaine : Publicité.

Définition : Réalisation définitive avant reAnglais : Artwork. production.

Écran de contrôle.

Voir Moniteur.

Écran témoin.

Voir Moniteur.

Égalisateur (n.m.)

Domaine : Audiovisuel/Son.

Définition : Ensemble électronique possédant un grand nombre de réglages de tonalité permettant de modeler, au gré de l'utilisateur, la courbe de réponse d'un amplificateur. 
Anglais : Equalizer.

Embargo (n.m.).

Domaine : Audiovisuel/Télévision, son.

Définition : Délai à respecter avant de diffuser une information ou un programme.

Note : Le terme anglais release ne doit pas être employé

Équilibreur (n.m.).

Synonyme : Équilibreur avant-arrière (n.m.).

Domaine : Audiovisuel/Son.

Définition : Sur un récepteur autoradio ou un amplificateur, dispositif de réglage permettant de doser le niveau acoustique entre les haut-parleurs avant

Anglais : Fader. et arrière.

Équilibreur avant-arrière.

Voir Équilibreur.

Esquisse.

Voir Crayonné.

Évanouissement (n.m.)

Domaine : Audiovisuel/Télévision, son.

Définition : Diminution momentanée de la puissance d'une onde radioélectrique au point de réception, pouvant aller jusAnglais : Fading qu'à sa disparition totale.

Fermeture en fondu. Voir Fondu (2).

Fondu (1) (n.m.).

Domaine : Audiovisuel/Son.

Définition : Abaissement volontaire et progressif du niveau du signal Anglais : Shunt. son jusqu'à l'annulation.

Fondu (2) (n.m.).

Synonymes: Fondu au noir (n.m.), Fermeture en fondu (n.f.).

Domaine : Audiovisuel/Cinéma, télévision.

Définition : Procédé par lequel on obtient la disparition progressive de 1'image.

Anglais : Fade out.

Fondu au noir.

Voir Fondu (2).

Fondu enchaîné (n.m.).

Domaine : Audiovisuel/Cinéma, télévision.

Définition : Combinaison simultanée d'un fondu et d'une ouverture en fondu.

Anglais : Cross fading.

Gros plan (n.m.) (Abréviation: G.P.).

Domaine : Audiovisuel/Cinéma, télévision, photo.

Définition : Cadrage d'une personne à la hauteur des épaules. Par ex- tension désigne toute prise de vue rapprochée d'un objet.

Anglais : Close up.

Groupiste (n.m.).

Domaine : Audiovisuel/Cinéma, télévision.

Définition : Personne responsable du fonctionnement d'un groupe électrogène.

Note : Le terme "groupman» ne doit pas être employé.

Images d'archives (n.f.pl.) (Abréviation : Archives [n.f.pl.]).

Domaine : Audiovisuel/Cinéma, télévision.

Définition : Images d'actualité de cinéma ou de télévision empruntées à des documents d'archives et insérées dans une cuvre postérieure de reportage ou de fiction.

Note : Se substitue à «plan d'archives».

Anglais : Stock-shot

Internégatif (n.m.)

Domaine : Audiovisuel/Cinéma, télévision.

Définition : Négatif tiré à partir d'une copie

Anglais

: Dupe. positive d'un négatif original.

Journalisme électronique (n.m.) (Abréviation: Jet [prononcé Jette]).

Synonyme : Reportage électronique (n.m.).

Domaine : Audiovisuel/Cinéma, télévision.

Définition : Reportage effectué avec des équipements vidéo suffisamment légers pour être portés par un ou deux opérateurs.

Anglais : Electronic news gathering (E.N.G.).

Maquette (n.f.).

Domaine : Publicité.

Définition : Projet permettant de donner l'aperçu le plus juste de ce que sera la réálisation.

Anglais : Advanced lay-out.

Média (n.m.).

Domaine : Communication (général).

Définition : Groupe de supports de même nature constituant un même moyen d'expression (presse, cinéma, télévision, radio, affiches, etc.).

Note : Le pluriel est médias.

Anglais : Media.

Médialogie (n.f.)

Domaine : Communication (général).

Dérivé : Médialogue (n.m. ou f.).

Définition : Étude des médias.

Médialogue (n.m. ou f.).

Domaine : Communication (général).

Définition : Spécialiste en médialogie. 
Mini-disque.

Voir Disque audio-numérique.

Moniteur (n,m.).

Synonyme : Écran témoin (n.m.), écran de contrôle (n.m.).

Domaine : Audiovisuel, télévision, vidéo.

Définition : Dispositif permettant de visionner des images vidéo notam-

Anglais : Monitor ment à des fins de contrôle.

Montage sur copie (n.m.)

Domaine : Audiovisuel/Vidéo.

Définition : Montage effectué à partir d'une bande vidéo copiée notamment sur une cassette.

Note : L'expression «montage off line» ne doit pas être employée.

Montage sur original (n.m.).

Domaine : Audiovisuel/Vidéo.

Définition : Montage effectué à partir d'une bande vidéo originale.

Note : L'expression "montage on line" ne doit pas être employée.

Monter sec.

Voir Sec.

Monter serré.

Voir Serré.

Opérateur de prises de vues.

Voir Cadreur.

Ouverture en fondu (n.f.).

Domaine : Audiovisuel/Cinéma, télévision.

Définition : Apparition volontaire et progres-

Anglais : Fade in sive de l'image.

Parrainage (n.m.)

Domaine : Publicité

Définition : Soutien donné à l'organisation d'une manifestation, financièrement ou au moyen de prestations de services, afin d'obtenir la contrepartie des effets publicitaires

Note : Le terme «sponsorisation" ne Anglais : Sponsoring.

Parrainer.

Voir Commanditer.

Perte de niveau (n.f.)

Domaine : Audiovisuel/Télévision, son, vidéo.

Définition : Affaiblissement brusque du Anglais : Drop-out. signal sonore ou visuel.

Piste d'asservissement (n.f.).

Domaine : Audiovisuel/Vidéo.

Définition : Piste sur la bande vidéo, qui permet au magnétoscope de s'asservir selon les indications qu'elle contient.
Anglais : Control track.

Plan d'archives.

Voir Images d'archives.

Plan-paquet.

Voir Plan produit.

Plan produit (n.m.).

Domaine : Publicité.

Définition : Plan permettant l'identification d'un produit.

Note : Se substitue à «plan-paquet».

Anglais : Pack-shot.

Plan serré (n.m.).

Domaine : Audiovisuel/Cinéma, télévision, photo.

Définition : Cadrage d'un personnage à la hauteur des épaules. Par extension, désigne toute prise de vue rapprochée d'un objet.

Anglais : Close up

Point de coupure (n.m.).

Domaine : Audiovisuel/Electronique.

Définition : Point de repère sur une courbe caractéristique de tube électronique ou de dispositif semi-conducteur, correspondant à l'arrêt du courant

Anglais : Cut off Dans une électrode donnée.

Porte-annonces (n.m.)

Domaine : Publicité

Définition : Recueil d'annonces publicitaires spécialement fabriqué pour les besoins d'une enquête et contenant l'annonce à étudier.

Anglais : Folder.

Post-enquête.

Voir Post-Test.

Post-test (n.m.)

Domaine : Publicité.

Définition : Évaluation ou mesure des effets d'un message publicitaire après sa diffusion.

Note : Se substitue à «post-enquête».

Anglais : Post-testing.

Pré-enquête.

Voir Pré-test.

Présentoir (n.m.)

Domaine : Publicité

Définition : Matériel de présentation et de distribution destiné à mettre en valeur un produit donné.

Anglais : Merchandiser.

Prêt-à-monter (n.m.)

Domaine : Audiovisuel/Électronique.

Définition : Ensemble électronique présenté en pièces détachées en vue d'être monté par l'utilisateur.

Note : On dit au pluriel des prêts-àmonter. 
Anglais : Kit.

Pré-test (n.m.).

Domaine : Publicité.

Définition : Évaluation ou mesure des effets supposés d'un message publicitaire avant sa diffusion.

Note : Se substitue à «pré-enquête».

Anglais : Pre-testing.

Promo.

Voir Bande vidéo promotionnelle.

Propre (n.m.)

Domaine : Audiovisuel/Vidéo.

Définition : Conducteur définitif d'un montage vidéo.

Anglais : Clean-list.

Publicitaire (n.m. ou adj.).

Domaine : Publicité.

Définition : - Professionnel de la publicité.

- Qui se rapporte à la publicité.

Note : Le terme «publiciste» ne doit pas être employé dans cette acception.

Anglais : Advertising man.

Publipostage (n.m.).

Domaine : Publicité.

Définition : Prospection, démarchage ou vente par voie postale, com-

Anglais : Mailing. portant souvent l'envoi d'échantillons.

Radiocassette (n.f.)

Domaine : Audiovisuel/Son.

Définition : Appareil combiné radiorécepteur, lecteur et enregistreur à cassette.

Anglais : Radiorecorder.

Régisseur de distribution (n.m.).

Domaine : Audiovisuel/Cinéma, télévision, publicité.

Définition : Responsable de la recherche des artistes et de l'attribution des rôles.

Anglais : Casting director.

Remue-méninges (n.m.).

Domaine : Publicité

Définition : Technique de groupe destinée à stimuler l'imagination des participants en vue de leur faire produire le maximum d'idées dans le minimum de temps.

Anglais : Brain-storming.

Reportage électronique.

Voir Journalisme électronique.

Resserrer un montage.

Voir Serré.

Retour en arrière (n.m.).

Domaine : Audiovisuel/Cinéma, télévision.

Définition : Rupture de la continuité chronologique d'une action et évocation d'une période liée

Anglais : Flash-back

Rotophaseur (n.m.).

Domaine : Audiovisuel/Son

Définition : Dispositif électronique simulant l'effet d'un haut-parleur tournant.

Anglais : Phasing.

Scanneur (n.m.).

Domaine : Audiovisuel (général).

Définition : Appareil ou dispositif capable de réaliser automatiquement le balayage continu de l'ensemble des canaux d'un récepteur.

Note : L'orthographe «scaneur» est admise.

Anglais : Scanner.

Scénarimage (n.m.).

Domaine : Audiovisuel/Cinéma, télévision, publicité.

Définition : Ensemble des dessins qui représentent, avant le tournage, le

Anglais : Story-board. film dans sa totalité.

Scripte (n.m. ou f.).

Domaine : Audiovisuel/Cinéma, télévision, publicité.

Définition : Responsable de la continuité de la réalisation et de la tenue des documents auprès du réalisateur d'un film ou d'une

Anglais : Script. émission.

Sec (adv.).

Domaine : Audiovisuel/Cinéma, télévision, son.

Définition : Adverbe utilisé dans les expressions:

- Couper sec : faire une coupure brusque et nette du plan sonore ou visuel.

- Monter sec: faire un montage de plans sonores ou visuels coupés de manière Anglais : Cut brusque et nette.

Sélectionné (adj.).

Domaine : Audiovisuel (général).

Définition : Retenu comme candidat pour un prix ou une distinction.

Note : Le terme «nominé » ne doit pas être employé.

Serré (adv.).

Domaine : Audiovisuel/Cinéma, télévision, son.

Définition : Adverbe utilisé dans 1'expression Monter serré : Obtenir un rythme vif et soutenu par 
une succession de plans sonores ou visuels.

Note : À rapprocher de l'expression

Resserrer un montage : Accélérer le rythme d'un montage en raccourcissant ou en supprimant certains Anglais : Cut lans ou séquences.

Silencieux (n.m.)

Domaine : Audiovisuel/Son.

Définition : Sur un syntoniseur ou sur un ampli-syntoniseur, dispositif électronique de suppression du bruit de fond apparaissant lors de la recherche d'une station.

Également, dispositif permettant d'affaiblir d'une valeur fixe le niveau de sortie d'un amplificateur.

Anglais : Muting.

Sonal (n.m.).

Domaine : Publicité, audiovisuel/télévision, son.

Définition : Message ou élément de message diffusé sur les médias audiovisuels, généralement fondé sur un thème musical répétitif et destiné à provoquer un Anglais : Jingle. reflexe de reconnaissance.

Sonorisation (n.f.).

Domaine : Audiovisuel/Son.

Définition : Ensemble des moyens permettant d'assurer la diffusion de la parole et de la musique dans un espace important.

Note

: L'abréviation «PA» de l'anglais «public address», ne doit pas être employée.

Anglais : Public address.

Souche (n.f.).

Synonyme : Bande-mère (n.f.).

Domaine : Audiovisuel/vidéo, son.

Définition : Bande magnétique originale de

Anglais : Master. montage.

Suramplificateur (n.m.)

Domaine : Audiovisuel/Son.

Définition : Amplificateur de puissance supplémentaire, notamment

Anglais : Booster pour un véhicule automobile.

Stylicien -ne (n.m. ou f.).

Domaine : Publicité, esthétique industrielle.

Définition : Spécialiste de la stylique.

Anglais : Designer.

Stylique (n.f.).

Dérivé : Stylicien -ne (n.m. ou f.).

Domaine : Publicité, esthétique industrielle.
Définition : Activité se rapportant à l'aspect extérieur d'un produit industriel en vue d'un résultat esthétique s'accordant aux impératifs fonctionnels et

Anglais : Design commerciaux.

Stylisme (n.m.)

Domaine : Publicité.

Définition : Activité professionnelle qui regroupe l'ameublement des décors, la recherche des accessoires et le choix des vètements en vue d'une présentation publicitaire.

Anglais : Styling elements.

Styliste (n.m. ou f.).

Domaine : Publicité.

Définition : Professionnel chargé du stylisme.

Note : Il peut être à la fois, ensemblier, accessoiriste et costumier.

Anglais : Stylist.

Syntonisateur.

voir Syntoniseur.

Syntoniseur (n.m.).

Domaine : Audiovisuel/Son.

Définition : Appareil électronique capable de convertir un signal radiofréquence reçu par une antenne en un signal audiofréquence de faible puissance.

Note : Le terme «syntonisateur» (n.m.) est employé au Canada.

Anglais : Tuner.

Télésouffieur (n.m.)

Domaine : Audiovisuel/Télévision.

Définition : Appareil permettant de lire un

Anglais : Teleprompter. texte face à la caméra.

Télévision à accès conditionnel (n.f.).

Domaine : Audiovisuel/Télévision.

Définition : Système de télévision dans lequel l'usager doit disposer d'une clé d'accès s'il veut recevoir certaines émissions.

Télévision à péage (n.f.).

Synonyme: Télévision payante (n.f.).

Domaine : Audiovisuel/Télévision.

Définition : Système de télévision dans lequel l'usager doit effectuer un paiement spécial s'il veut recevoir certaines émissions.

Anglais : $P a y-T V$.

Télévision payante.

Voir Télévision à péage

Test aveugle (n.m.).

Domaine : Publicité. 
Définition : Test de comparaison entre deux ou plusieurs produits anonymement présentés.

Anglais : Blind test.

Test de porte-annonces (n.m.)

Domaine : Publicité

Définition : Mesure de l'impact, de la compréhension et de l'acceptabilité d'une annonce insérée dans un porte-annonces.

Anglais : Folder-test.

Test d'évaluation (n.m.).

Domaine : Publicité.

Définition : Procédure destinée à déterminer les réactions d'une clientèle visée face à un message ou à une série de messages publicitaires.

Anglais : Copy testing.

Test du lendemain (n.m.). (Abréviation : T.D.L.)

Domaine : Publicité.

Définition : Mesure de l'impact d'un message publicitaire vingt-quatre heures après sa diffusion.

Anglais : Day after recall (D.A.R.).

Test sur tirage équifractionné (n.m.).

Domaine : Publicité.

Définition : Technique destinée à mesurer les réponses relatives à deux annonces différentes, en insérant chacune d'elles dans la moitié des exemplaires d'un support de presse Anglais : Split run test. également distribués.

Tirage équifractionné (n.m.).

Domaine : Publicité.

Définition : Tirage fractionné d'une revue ou d'un journal dont la distribution rigoureusement égale permet la comparaison de deux annonces différentes Anglais : Split run. pour un même produit.

Traitement acoustique (n.m.).

Domaine : Audiovisuel/Son.

Définition : Utilisation de matériaux spécifiques permettant de rendre un local agréable du point de vue auditif.

Transcrire (v.tr.).

Domaine : Audiovisuel/Cinéma, télévision.

Définition : Relever par écrit les paroles d'une bande sonore.

Note : 1. Le terme «scripter" ne doit pas être employé.

2. Le terme «décrypter» ne doit pas être employé dans cette acception.

Travail de terrain (n.m.).

Domaine : Publicité.
Définition : Phase d'enregistrement de données, réalisée dans un processus d'enquête auprès d'une catégorie de population pré-désignée.

Anglais : Field work

Très gros plan (n.m.). (Abréviation: T.G.P.).

Domaine : Audiovisuel/Cinéma, télévision, photo.

Définition : Cadrage d'un détail du visage. Par extension, désigne toute prise de vue très rapprochée d'un objet.

Anglais : Big close up.

Verrouilleur de synchronisation (n.m.). (Abréviation: Verrouilleur [n.m.].)

Domaine : Audiovisuel/Vidéo.

Définition : Dispositif permettant d'asservir le générateur de synchronisation sur un signal extérieur.

Anglais : Gen-lock.

Voix dans le champ (n.f.).

Domaine : Audiovisuel/Cinéma, télévision.

Définition : Voix d'une personne présente à l'écran.

Note : L'expression «Voix in" ne doit pas être employée.

Voix hors champ (n.f.).

Domaine : Audiovisuel/Cinéma, télévision.

Définition : Voix d'une personne absente à l'écran.

Note : L'expression «Voix off $»$ ne doit pas être employée.

Annexe II

Index alphabétique des termes à éviter.

Termes à éviter. Termes recommandés

Advanced lay-out. Maquette.

Advertising man. Publicitaire.

Air print. Copie d'antenne.

Amplificateur de public Amplificateur de sonori-

address.

Ampli-tuner.

Artwork.

Audio-video-split.

sation.

Ampli-syntoniseur.

Document.

Big close up.

Blind test.

Booster.

Désynchronisation ponctuelle.

Très gros plan (T.G.P.).

Test aveugle.

Suramplificateur.

Brain-storming.

Cableman.

Cameraman.

Casting, cast.

Casting director.

Catching.

Clean-list.

Close up.
Remue-méninges.

Câbliste.

Cadreur, opérateur de prises de vues.

Distribution artistique.

Régisseur de distribution.

Accroche.

Propre.

Gros plan (G.P.), plan serré. 
Termes à éviter.

Control track.

Copy platform.

Copy testing.

Cross fading.

Cut.

Cut off.

Day after recall

(D.A.R.)

Décrypter (

Design.

Designer.

Display.

Drive-in cinema.

Drop-out.

Dupe.

Electronic news gathering (E.N.G.).

Equalizer

Fade in.

Fade out.

Fader.

Fading.

Field work.

Flash-back.

Folder.

Folder-test.

Gen-lock.

Groupman.

Jingle.

Kit.

Loudness.

Mailing.

Master.

Media.

Merchandiser.

Monitor.

Montage off line

Montage on line.

Muting.

Nominé.
Termes recommandés.

Disque audionumérique, mini-disque.

Piste d'asservissement.

Base de campagne.

Test d'évaluation.

Fondu enchaîné.

Sec, serré.

Point de coupure.

Test du lendemain

(T.D.L.).

Transcrire

Stylique.

Stylicien

Carton publicitaire, carton.

Ciné-parc.

Perte de niveau.

Internégatif.

Journalisme électro-

nique de télévision (jet), reportage électronique.

Égaliseur.

Ouverture en fondu.

Fondu, fondu au noir, fermeture en fondu.

Équilibreur, équilibreur avant-arrière.

Évanouissement.

Travail de terrain.

Retour en arrière.

Porte-annonces.

Test de porte-annonces.

Verrouilleur de synchronisation, verrouilleur.

Groupiste.

Sonal.

Prêt-à-monter.

Contour.

Publipostage.

Souche, bande-mère.

Média.

Présentoir.

Moniteur, écran témoin, écran de contrôle.

Montage sur copie.

Montage sur original.

Silencieux.

Sélectionné.
Termes à éviter.

Overlap.

Package.

Pack-shot.

Pay-TV.

Phasing.

Post-testing.

Pre-testing.

Public address amplifier, public address (P.A.).

Publiciste (au sens de). Publicitaire.

Radiorecorder.

Receiver.

Release.

Release print.

Rough lay-out, rough.

Scanner.

Script.

Scripter.

Shunt.

Simple lay-out.

Split run.

Split run test.

Sponsor.

Sponsor (to).

Sponsoring.

Sponsorisation.

Sponsoriser.

Stock-shot.

Story-board.

Styling elements.

Stylist.

Teaser.

Teasing,

Teleprompter.

Time-code.

Tuner.

Video-clip.

Video tape.

Voix in.

Voix off.

Walkman.
Termes recommandés.

Débordement.

Achat groupé.

Plan-produit.

Télévision à péage, télévision payante.

Phaseur.

Post-test.

Pré-test.

Sonorisation

Radiocassette.

Ampli-syntoniseur.

Embargo.

Copie d'antenne.

Crayonné, esquisse.

Scanneur.

Scripte.

Transcrire.

Fondu.

Crayonné, esquisse.

Tirage équifractionné.

Test sur tirage équifractionné.

Commanditaire.

Commanditer, parrainer.

Parrainage.

Pairrainage.

Commanditer, parrainer.

Images d'archives, archives.

Scénarimage.

Stylisme.

Styliste.

Aguiche.

Aguichage.

Télésouffleur.

Code temporel.

Syntoniseur.

Bande vidéo promotionnelle, bande promo, promo.

Bande vidéo.

Voix dans le champ.

Voix hors champ.

Baladeur. 